\title{
Darboux vector and stress analysis of Winternitz frame
}

\author{
Yilmaz Tuncer ${ }^{1}$ and Huseyin Kocayigit ${ }^{2}$ \\ ${ }^{1}$ Usak University, Science and Art Faculty, Department of Mathematics, Usak, Turkey \\ ${ }^{2}$ Manisa Celal Bayar University, Science and Art Faculty,Department of Mathematics, Manisa, Turkey
}

Received: 6 August 2018, Accepted: 29 October 2018

Published online: 30 December 2018.

\begin{abstract}
In a set of points that corresponds a vector of vector space constructed on a field is called an affine space associate with vector space. We denote as affine 3-space associated with. The first written sources that can be achieved about affine space curve theory are based on the 1890's when Ernesto Cesàro and Die Schon von Pirondini lived period. From that years to 2000's there are a some affine frames used in curve theory. One of them is Winternitz frame which is in equi-affine transformation group. The grup of affine motions special linear transformation consist of volume preserving linear transformations denoted by and comprising diffeomorphisms of that preserve some important invariants such curvatures that in curve theory as well. In this study, we separated the matrix representing affine frame as symmetric and antismmetric parts by using matrix demonstration of the Winternitz frame of a curve given in affine 3 -space. By making use of antisymmetric part, we obtained the angular velocity vector which is also known as Darboux vector and then we expressed it in the form of linear sum of affine Frenet vectors. On the other hand, by making use of symmetric part, we obtained the normal stresses and shear stress components of the stress on the frame of the curve in terms of the affine curvature and affine torsion. Thus we had the opportunity to be able to explane the distinctive geometric features of the affine curvature and affin torsion. Lastly, we made stress analysis of a curve with constant affine curvature and affine torsion in affine 3-space as an example.
\end{abstract}

Keywords: Winternitz frame, Darboux vector, stress analysis.

\section{Introduction}

The grup of affine motions special linear transformation namely the group of equi-affine or unimodular transformations consists of volume preserving $\left(\operatorname{det}\left(a_{j k}\right)=1\right)$ linear transformations together with translation such that

$$
x_{j}^{*}=\sum_{k=1}^{3} a_{j k} x_{k}+c_{j} \quad j=1,2,3
$$

This transformations group denoted by $A S L(3, I R):=S L(3, I R) \times I R^{3}$ and comprising diffeomorphisms of $I R^{3}$ that preserve some important invariants such curvatures that in curve theory as well. An equi-affine group is also called a Euclidean group.

A set of points that corresponds a vector of vector space constructed on a field is called an affine space associate with vector space. We denote $A_{3}$ as affine 3-space associated with $I R^{3}$. Let

$$
\alpha: J \longrightarrow A_{3}
$$


be a curve in $A_{3}$, where $J=\left(t_{1}, t_{2}\right) \subset I R$ is a fixed open interval. Regularity of a curve in $A_{3}$ is defined as $|\dot{\alpha} \ddot{\alpha} \dddot{\alpha}| \neq 0$ on $J$, where $\dot{\alpha}=d \alpha / d t$, etc. Then with $\alpha$ we may associate the invariant parameter

$$
\sigma(t)=\int_{t_{1}}^{t}|\dot{\alpha} \ddot{\alpha} \ddot{\alpha}|^{1 / 6} d t \quad(\cdot=d / d t)
$$

which is called the affine arc length of $\alpha$. The coordinates of a curve are given by three linearly independent solutions of the equations

$$
\alpha^{(i v)}(s)+v(s) \alpha^{\prime \prime}(s)+\omega(s) \alpha^{\prime}(s)=0
$$

under the condition

$$
\left|\alpha^{\prime}(s), \alpha^{\prime \prime}(s), \alpha^{\prime \prime \prime}(s)\right|=1
$$

where $v(s)$ and $\omega(s)$ denote the affine curvatures. Some remarkable geometrical definitions of $\alpha^{\prime \prime}(s)$ and $v(s)$ were discovered by R. Weizenböck and G. Sannia and L. Berwald. E. Salkowski, W.Shells and A. Winternitz studied and gave some special features both planar and space curves by using equi-affine frame[1,2,9,4,5]. According to the analogy of Euclidean space theory, it would prefer to take the vectors $\left\{\alpha^{\prime}(s), \alpha^{\prime \prime}(s), \alpha^{\prime \prime \prime}(s)\right\}$ for the moving triad of a curve $\alpha(s)$ in affine space, and then $v(s), \omega(s)$ for natural equations. Since it cannot conclude therefrom that $v(s)$ and $\omega(s)$ are invariants of the lowermost order determined by the curve, this is not desirable. A. Winternitz is the first mathematician who pointed out this fact and then constructed a moving frame, the Winternitz frame, of the lowermost or the 4th order $[6,1,5]$.

We can decompose any square matrix $Q$ uniquely as

$$
Q=\frac{Q+Q^{t}}{2}+\frac{Q-Q^{t}}{2}
$$

with symmetric part $\frac{Q+Q^{t}}{2}$ and with antisymmetric part $\frac{Q-Q^{t}}{2}$. If $Q$ is anti-symmetric then symmetric part is zero matrix. According to A.Cayley's transformation

$$
R=\left[I+\frac{Q-Q^{t}}{2}\right]^{-1}\left[I-\frac{Q-Q^{t}}{2}\right]
$$

is a orthogonal (rotation) matrix which is $|R|=+1[7]$. The stress tensor is a square symmetric matrix. In 2-dimensional space, stress matrix is given

$$
\Psi=\left[\begin{array}{cc}
\sigma_{X} & \sigma_{X Y} \\
\sigma_{X Y} & \sigma_{Y}
\end{array}\right]
$$

according to $\{X, Y\}$ coordinate system. The matrix $\Psi$ consist of the three stress components $\sigma_{X}, \sigma_{Y}$ and $\sigma_{X Y}$ which means stresses on $X$, on $Y$ directions, and stresses on $\{X, Y\}$ planes respectively. Similarly, the matrix $\widetilde{\Psi}$

$$
\widetilde{\Psi}=\left[\begin{array}{ccc}
\sigma_{X} & \sigma_{X Y} & \sigma_{X Z} \\
\sigma_{X Y} & \sigma_{Y} & \sigma_{Y Z} \\
\sigma_{X Z} & \sigma_{Y Z} & \sigma_{Z}
\end{array}\right]
$$

consist of the six stress components $\sigma_{X}, \sigma_{Y}, \sigma_{Z}, \sigma_{X Y}, \sigma_{X Z}$ and $\sigma_{Y Z}$ which means stresses parallel to $X$, parallel to $Y$, parallel to $Z$ directions, and shear stress in $Y$ direction on $Y Z$ plane normal to $X$-axis, shear stress in $Z$ direction on $Y Z$ plane normal to $X$-axis, shear stress in $Z$ direction on $X Z$ plane normal to $Y$-axis respectively[8,9]. 


\section{Winternitz's affine frame and Darboux vector}

A $C^{\infty}$ map $\alpha$ from an interval $I$ to $I R^{2}$ is called an equiaffine plane curve in $I R^{2}$ if $\left|\alpha^{\prime}(s) \alpha^{\prime \prime}(s)\right| \neq 0$, and $\alpha$ is said to be parameterized by equiaffine arclength

parameter if $\left|\alpha^{\prime}(s) \alpha^{\prime \prime}(s)\right|=1$ for all $s \in I$. For an equiaffine plane curve parameterized by equiaffine arc-length parameter, the invariant equiaffine curvature defined by

$$
k(s)=\left|\alpha^{\prime \prime}(s) \alpha^{\prime \prime \prime}(s)\right|
$$

so for $T(s):=\alpha^{\prime}(s)$ and $N(s):=\alpha^{\prime \prime}(s)$ which are called tangent and affine normal vectors, we have

$$
\left[\begin{array}{c}
T^{\prime}(s) \\
N^{\prime}(s)
\end{array}\right]=\left[\begin{array}{cc}
0 & 1 \\
-k(s) & 0
\end{array}\right]\left[\begin{array}{l}
T(s) \\
N(s)
\end{array}\right]
$$

We can decompose $q$ uniquely as $q=p+w$ with symmetric part

$$
p=\frac{1}{2}\left[\begin{array}{cc}
0 & 1-k(s) \\
1-k(s) & 0
\end{array}\right]
$$

which means stress part and with antisymmetric part

$$
w=\frac{1}{2}\left[\begin{array}{cc}
0 & 1+k(s) \\
-(1+k(s)) & 0
\end{array}\right] .
$$

Also, we can find the rotation matrix $r$ of equi-affine frame motion such as

$$
r=\frac{1}{(k+1)^{2}+4}\left[\begin{array}{cc}
-(k+1)^{2}+4 & -4(k+1) \\
4(k+1) & -(k+1)^{2}+4
\end{array}\right]
$$

which is $|r|=+1$.

The matrix $p$ consist of the three stress components $\sigma_{T}=\sigma_{N}=0$ and $\sigma_{T N}=(1-k(s)) / 2$ which means stresses on tangent, on normal directions, and stresses on $\{T, N\}$ plane, respectively. The two principal stresses are roots of $\left|\sigma I_{2}-p\right|=0$

$$
\sigma_{1,2}= \pm \frac{|1-k(s)|}{2}
$$

and corresponding eigen vectors

$$
v_{1,2}=(\mp 1,1) \in \operatorname{sp}\{T, N\}
$$

according to $\{T, N\}$. Thus we can give the following remark.

Remark. Throughout the planar equi-affine frame motion there are no sresses on tangent and normal directions but there are two principal stresses $\sigma_{1}=\frac{|1-k(s)|}{2}$ and $\sigma_{2}=-\frac{|1-k(s)|}{2}$ acts on principal axises $v_{1}(s)=-T(s)+N(s)$ and $v_{2}(s)=$ $T(s)+N(s)$ respectively. Additionally, throughout the motion there is shear stress on $\{T, N\}$ plane with the magnitute $\frac{1-k(s)}{2}$.

K. Nomizu, and T. Sasaki [11], obtained some plane curves with constant curvature and gave the following important theorem.

Theorem 1. An equiaffine plane curve $\alpha(s)$ with constant $k(s):=k$ is equiaffinely equivalent to one of the following curve 
(i) If $k=0$,

$$
\alpha(s)=\left(s, \frac{1}{2} s^{2}\right)
$$

that is a parabola $y=\frac{1}{2} x^{2}$,

(ii) If $k>0$,

$$
\alpha(s)=\left(k^{-1 / 2} \sin \left(k^{-1 / 2} s\right),-k^{-1} \cos \left(k^{-1 / 2} s\right)\right)
$$

that is an ellipse $k x^{2}+k y^{2}=1$

(iii) If $k<0$,

$$
\alpha(s)=\left((-k)^{-1 / 2} \sinh \left((-k)^{-1 / 2} s\right),(-k)^{-1} \cosh \left((-k)^{-1 / 2} s\right)\right)
$$

that is a hyperbola $k x^{2}-k y^{2}=1$.

If $k(s)=1$ then two principal shear strees are zero. Shear stress is zero for an ellips $x^{2}+y^{2}=1$ and $\pm 1 / 2$ for a parabola.

Onthe other hand, let $\alpha(s)$ be regular curve in affine 3-space with the affine arclenght parameter $s$. The vectors $\alpha^{\prime}(s), \alpha^{\prime \prime}(s)$ and $\alpha^{\prime \prime \prime}(s)$ are called tangent, affine normal and binormal vectors respectively, and the planes $s p\left\{\alpha^{\prime}(s), \alpha^{\prime \prime}(s)\right\}, \operatorname{sp}\left\{\alpha^{\prime}(s), \alpha^{\prime \prime \prime}(s)\right\}$ and $\operatorname{sp}\left\{\alpha^{\prime \prime}(s), \alpha^{\prime \prime \prime}(s)\right\}$ are called osculating, rectifying and normal planes of the curve $\alpha(s)$. Thus the frame given in the matrix form as $\alpha^{\prime}(s)=T(s), N(s)=\alpha^{\prime \prime}(s), B(s)=\alpha^{\prime \prime \prime}(s)+\frac{\kappa(s)}{4} \alpha^{\prime}(s)$ and $k_{1}(s)=\frac{-\kappa(s)}{4}, k_{2}(s)=\frac{\kappa^{\prime}(s)}{4}-\tau(s)$ where where $\kappa(s)$ and $\tau(s)$ are called affine curvature and affine torsion which are given

$$
\begin{aligned}
& \kappa(s)=\left|\begin{array}{lll}
\alpha^{\prime}(s) & \alpha^{\prime \prime \prime}(s) & \alpha^{(i v)}(s)
\end{array}\right| \\
& \tau(s)=-\left|\alpha^{\prime \prime}(s) \alpha^{\prime \prime \prime}(s) \alpha^{(i v)}(s)\right|
\end{aligned}
$$

and $k_{1}(s), k_{2}(s)$ are called first and secod affine curvatures [1].

$$
\left[\begin{array}{l}
T^{\prime}(s) \\
N^{\prime}(s) \\
B^{\prime}(s)
\end{array}\right]=\left[\begin{array}{ccc}
0 & 1 & 0 \\
k_{1}(s) & 0 & 1 \\
k_{2}(s) & 3 k_{1}(s) & 0
\end{array}\right]\left[\begin{array}{c}
T(s) \\
N(s) \\
B(s)
\end{array}\right]
$$

is called Winternitz frame (also called equi-affine frame for $C^{5}$-curves), as follows For

$$
Q=\left[\begin{array}{ccc}
0 & 1 & 0 \\
k_{1}(s) & 0 & 1 \\
k_{2}(s) & 3 k_{1}(s) & 0
\end{array}\right]
$$

we can decompose $Q$ uniquely as $Q=P+W$ with symmetric part

$$
P=\left[\begin{array}{ccc}
0 & \left(1+k_{1}(s)\right) / 2 & k_{2}(s) / 2 \\
\left(1+k_{1}(s)\right) / 2 & 0 & \left(1+3 k_{1}(s)\right) / 2 \\
k_{2}(s) / 2 & \left(1+3 k_{1}(s)\right) / 2 & 0
\end{array}\right]
$$

and with antisymmetric part

$$
W=\left[\begin{array}{ccc}
0 & \left(1-k_{1}(s)\right) / 2 & -k_{2}(s) / 2 \\
-\left(1-k_{1}(s)\right) / 2 & 0 & \left(1-3 k_{1}(s)\right) / 2 \\
k_{2}(s) / 2 & -\left(1-3 k_{1}(s)\right) / 2 & 0
\end{array}\right]
$$


Also, by using 4 we can find the rotation matrix $R$ of Winternitz affine frame motion such as

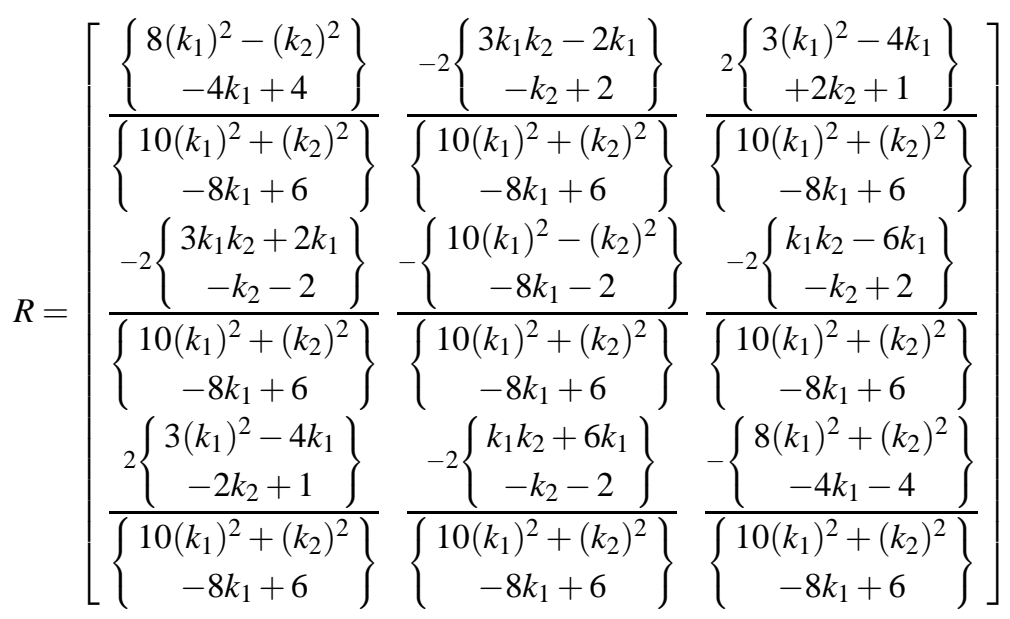

which is $|R|=+1$.

Theorem 2. For any nondegenerate space curve $\alpha$, instantaneous rotatinon matrix is

$$
R=\frac{1}{\left\{10\left(k_{1}\right)^{2}+\left(k_{2}\right)^{2}-8 k_{1}+6\right\}}\left[a_{i j}\right]
$$

where

$$
\begin{aligned}
& a_{11}=\left\{8\left(k_{1}\right)^{2}-\left(k_{2}\right)^{2}-4 k_{1}+4\right\}, \\
& a_{12}=-2\left\{3 k_{1} k_{2}+2 k_{1}-k_{2}-2\right\}, \\
& a_{13}=2\left\{3\left(k_{1}\right)^{2}-4 k_{1}-2 k_{2}+1\right\}, \\
& a_{21}=-2\left\{3 k_{1} k_{2}-2 k_{1}-k_{2}+2\right\}, \\
& a_{22}=-\left\{10\left(k_{1}\right)^{2}-\left(k_{2}\right)^{2}-8 k_{1}-2\right\}, \\
& a_{23}=-2\left\{k_{1} k_{2}+6 k_{1}-k_{2}-2\right\}, \\
& a_{31}=2\left\{3\left(k_{1}\right)^{2}-4 k_{1}+2 k_{2}+1\right\}, \\
& a_{32}=-2\left\{k_{1} k_{2}-6 k_{1}-k_{2}+2\right\}, \\
& a_{33}=-\left\{8\left(k_{1}\right)^{2}+\left(k_{2}\right)^{2}-4 k_{1}-4\right\},
\end{aligned}
$$

and instantaneous rotation vector is

$$
D=\frac{\left(1-3 k_{1}(s)\right)}{2} T(s)+\frac{k_{2}(s)}{2} N(s)-\frac{\left(1-k_{1}(s)\right)}{2} B(s) .
$$

On the other hand, the matrix $P$ consist of the six stress components $\sigma_{T}=\sigma_{N}=\sigma_{B}=0, \sigma_{T N}=\frac{1+k_{1}}{2}, \sigma_{T B}=\frac{k_{2}}{2}$ and $\sigma_{N B}=\frac{1+3 k_{1}}{2}$ which means stresses parallel to tangent, parallel to normal, parallel to binormal directions, and shear stress in $N$ direction on osculating plane normal to $T$-axis, shear stress in $B$ direction on rectifying plane normal to $T$-axis, shear stress in $B$ direction on normal plane normal to $N$-axis respectively[8,9]. The three prinicipal stresses are the eigen values of $P$ which are the roots of $\left|\sigma I_{3}-P\right|=0$,

$$
\sigma^{3}+\psi \sigma-\varphi=0
$$


where $\psi=-\frac{1}{4}\left\{\left(k_{2}\right)^{2}+\left(1+3 k_{1}\right)^{2}+\left(1+k_{1}\right)^{2}\right\}, \varphi=\frac{k_{2}\left(1+k_{1}\right)\left(1+3 k_{1}\right)}{4}$. Let the roots be $\widetilde{\sigma}_{1}, \widetilde{\sigma}_{2}$ and $\widetilde{\sigma}_{3}$ then three principal stresses are

$$
\begin{aligned}
& \widetilde{\sigma}_{1}=\frac{\Gamma^{2}-12 \psi}{6 \Gamma} \\
& \widetilde{\sigma}_{2}=\frac{-\Gamma^{2}+12 \psi+I \sqrt{3}\left\{\Gamma^{2}+12 \psi\right\}}{12 \Gamma} \\
& \widetilde{\sigma}_{3}=\frac{-\Gamma^{2}+12 \psi-I \sqrt{3}\left\{\Gamma^{2}+12 \psi\right\}}{12 \Gamma}
\end{aligned}
$$

where $\Gamma=\left\{108 \varphi+12 \sqrt{12 \psi^{3}+81 \varphi^{2}}\right\}^{1 / 3}$. Thus we can give the following theorem.

Theorem 3. Throughout the Winternitz affine frenet motion there are no stresses on tangent, on normal and on binormal directions but there are three principal stresses $\tilde{\sigma}_{i}$ acts on corresponding principal axises. Additionally, throughout the motion there are shear stresses $\sigma_{T N}=\frac{1+k_{1}}{2}, \sigma_{T B}=\frac{k_{2}}{2}$ and $\sigma_{N B}=\frac{1+3 k_{1}}{2}$ on osculating plane, on rectifying plane, on normal plane, respectively.

\section{Competing interests}

The authors declare that they have no competing interests.

\section{Authors' contributions}

All authors have contributed to all parts of the article. All authors read and approved the final manuscript.

\section{References}

[1] Su, B.: Affine Differential Geometry, Science Press, Beijing, China, 1983.

[2] Cesàro, E.: Lezioni di Geometria Intrinseca, Napoli, Italy, 1896.

[3] E. Salkowski, E. Schells, W.: Allgemeine Theorie der Kurven doppelter Krümmung, Leipzig und Berlin, 1914.

[4] Hu, N.: Centro-affine space curves with constant curvatures and homogeneous surfaces, J. Geom. 102, 103-104, (2011).

[5] Blaschke, W.: Differential geometrie II, Verlag von Julius springer, Berlin, 1923.

[6] Su, B.: Some Classes of Curves in The Affine space, Tohoku Math. Journ. 31,283-291, (1929).

[7] Cayley, A.: About the algebraic structure of the orthogonal group and the other classical groups in a field of characteristic zero or a prime characteristic, J. Reine Angew.Math., 32, (1846).

[8] Ferdinand, P.B. Russell, E. Johnson, Jr.: Mechanics of Materials, Second Edition, McGraw-Hill, Inc, 1992.

[9] James M.G. Stephen P.T.: Mechanics of Materials, Third Edition, PWS-KENT Publishing Company, Boston, 1990.

[10] Lai, W.M. Rubin, D. Krempl, E.: Introduction to continuum mechanics, Butterworth-Heinemann/Elsevier, Amsterdam , Boston, 2010.

[11] Nomizu, K. Sasaki, T.: A new model of unimodular-affinely homogeneous surfaces, Manuscripta math. 73, 39-44 (1991). 